\title{
Study of the gas driven water rivulet flow regimes in the minichannels
}

\author{
Olga Svetlichnaya ${ }^{1,2}$, Vyacheslav Cheverda ${ }^{2,3, *}$ \\ ${ }^{1}$ Novosibirsk State University, 630090 Novosibirsk, Russia \\ ${ }^{2}$ Kutateladze Institute of Thermophysics, 630090 Novosibirsk, Russia \\ ${ }^{3}$ National Research Tomsk Polytechnic University, 634050 Tomsk, Russia
}

\begin{abstract}
The paper deals with the experimental study of the gas (nitrogen) driven rivulet flow regimes of water outflowing from the nozzle with a width of $10 \mathrm{~mm}$ into minichannel with the height of $1.6 \pm 0.02 \mathrm{~mm}$ and a width of $30 \mathrm{~mm}$. The authors revealed a new flow regime - namely "rivulet bridge" whose occurrence is attributed to the influence of relatively large static wetting angle of water on the flow regimes. The paper presents a map of the various rivulet flow regimes characterized by different surface geometry and types of surface deformations.
\end{abstract}

\section{Introduction}

Against the background of the rapid development of electronics (reduction in the characteristic size of transistors) there is a need for effective cooling system that can be used in the aircraft industry (braking systems, microelectronics, etc.), automotive industry (power electronics in inverters, braking system, etc.) and in other industries.

A liquid film flow refers to a separate flow in which the fluid flows over the entire surface of the substrate. At a rivulet flow only a portion of the substrate is wetted by liquid, while the other part remains dry. Due to abnormally high heat transfer coefficient in the area of three phase contact line $[1,2]$, a rivulet flow has high cooling quality, since it is a particular case of the film flow with a contact line bounding the film from both sides, the line, where three phases come into contact. The fluid viscosity is significantly higher than gas viscosity. Due to this circumstance the use of rivulet flow will provide reduced resistance of the two-phase flow, as well as reduced pressure drop along the channel, and consequently, will reduce energy input for pumping of two-phase mixture through a microchannel. Despite the increased interest to this kind of problems, a number of available experimental [3,4] and theoretical works [5] is quite small and extremely insufficient for comprehension of the physics of the processes associated with the heat transfer in gas driven rivulet flow of water in the minichannel._Following types of the rivulet flows over inclined surfaces are currently available in the literature: drip flow, straight flow, meandrine flow, flow without waves and with 2D waves, 3D and soliton-type wave flows, as well as swirling flow.

\footnotetext{
${ }^{*}$ Corresponding author: slava.cheverda@gmail.com
} 
The aim of this work is constructing flow regime map of the gas driven isothermal rivulet flow of water in the minichannel. The results obtained will be used in the further development of the cooling system.

\section{Experimental setup}

Experimental studies were conducted employing setup, which is presented in Fig.1. The test bench for laboratory experiments consists of the following components: gas supply system; fluid delivery system; the system for maintaining a predetermined pressure in the test section and a regenerative system; the power supply system (not shown); a personal computer (PC-1) to control the experiment and collect experimental data; a personal computer (PC-2) for recording images from high-speed video camera; the water thermo stabilization system; the data collecting system; the optical system; the replaceable test section with the temperature-controlled substrate of the minichannel (from 10 to $100{ }^{\circ} \mathrm{C}$ ).

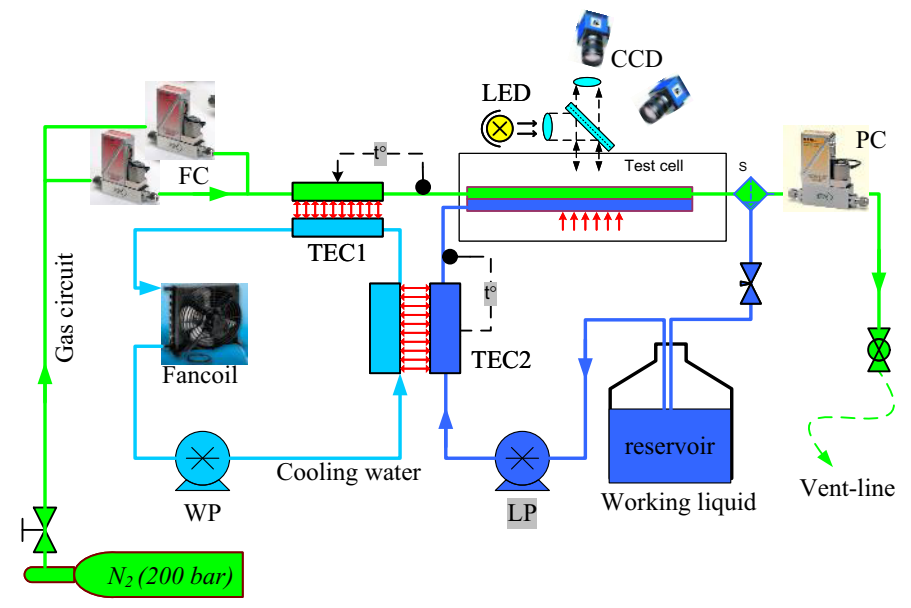

Fig. 1. The sketch of experimental setup for studying the nitrogen driven isothermal rivulet flow regimes of the working fluid (water) in a horizontal minichannel.

The frame (5) and cover (4) made of textolite serve the basis of the test section (Fig. 2a). Initial temperature of the liquid (11) and gas (12) at the inlet of minichannel, as well as temperature of the substrate (3) are varied within the range from 20 to $90{ }^{\circ} \mathrm{C}$ with an accuracy of $0.2^{\circ} \mathrm{C}$ using Peltier elements (8) which are cooled by water heat exchanger (9). The inlet temperatures of the gas and liquid as well as outlet temperature of the mixture, the temperature of the substrate (2) and the ambient temperature are measured by thermal sensors.

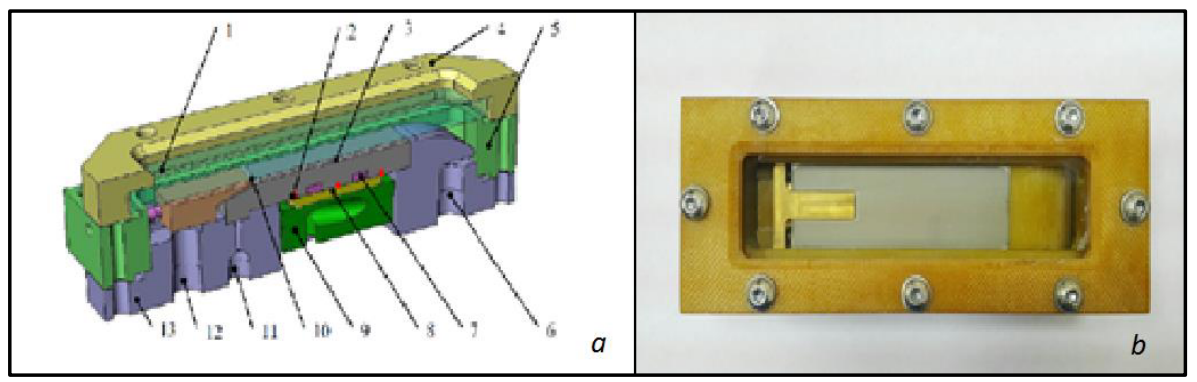

Fig. 2. Sketch (a) and photograph (2) of the test section. 
The test section is closed on top by sealed cover provided with an optical glass (1), thereby forming a minichannel with a width of $30 \mathrm{~mm}$ and a height of $1.61 \pm 0.02 \mathrm{~mm}$. Liquid is fed to the buffer chamber of the test section, passes through the nozzle between the brass plate (a blade) and the substrate and gets into the minichannel. The nozzle width for rivulet flow is equal to $10 \mathrm{~mm}$. Gas (nitrogen) is fed into the test section under pressure through the nozzle (14) and drives the fluid rivulet along a rough substrate made of stainless steel (3). Further, mixture of water and nitrogen is released through the orifice (6).

Employing DSA-100 system and using sessile drop method (see Fig. 3) we measured the static wetting angle which amounted to $114.4 \pm 3^{\circ}$.

Also, we measured surface roughness for a better understanding of the interaction between the substrate and the liquid. The arithmetic average of the absolute values of the profile deviations within the basic length ( $\mathrm{Ra}$ ) was $0.458 \mu \mathrm{m}$ (Fig.4.). The surface was processed with abrasive M-40 and the roughness was measured by the TR-200 instrument.

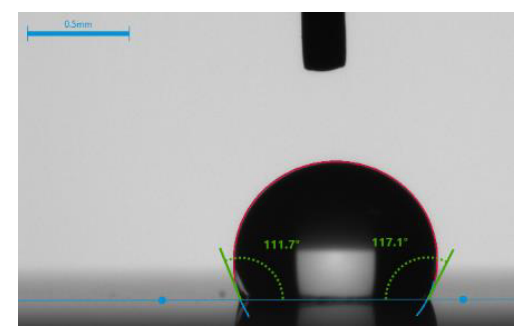

Fig. 3. Photograph of the water drop on a substrate.

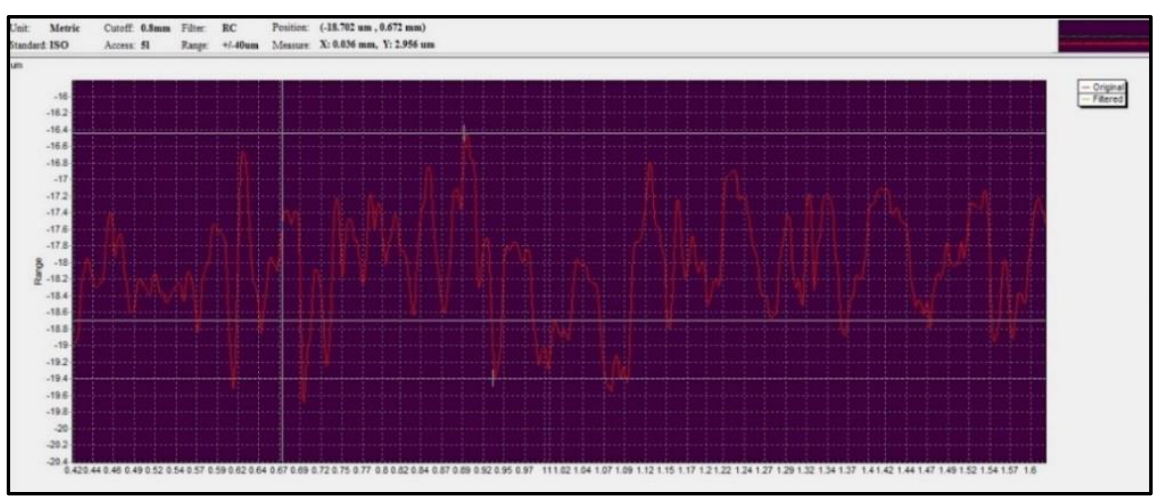

Fig. 4. The substrate surface roughness in a certain direction.

\section{Results and Discussion}

We have constructed regime map for variety of gas driven rivulet flows of water in the minichannel (Fig. 5). At that, we discovered a new regime, which has not previously been observed for the FC-72 fluid flow, namely "rivulet bridge". When flowing in this regime, the rivulet does not contact the side walls of the channel, though occupies its entire height. Moreover, we observed different variations in the "rivulet bridge" flow regime: pulsating flow regime, straight flow regime, divergent flow regime, and tapered flow regime. The emergence of a new flow regime is associated with the fact that the static wetting angle of water is much higher than that in the fluorinert fluid FC-72. It means that the height of the water rivulet when driven by the gas in the minichannel is much greater than that of FC-72 
fluid. Hence, we can conclude that the static wetting angle significantly affects the rivulet flow regimes.

We have also observed the regimes, where the geometry of the rivulet has been previously found for the FC-72 fluid flow in the minichannel. These are straight, tapering, and pulsating flow regimes.

For better visualization we have also constructed a map representing various surface deformation regimes (Fig. 6). At that, we observed wave generation regime, already familiar to us from the experiments with FC-72 fluid, as well as a new braiding regime (Fig. 8) that is caused by a larger surface tension coefficient.

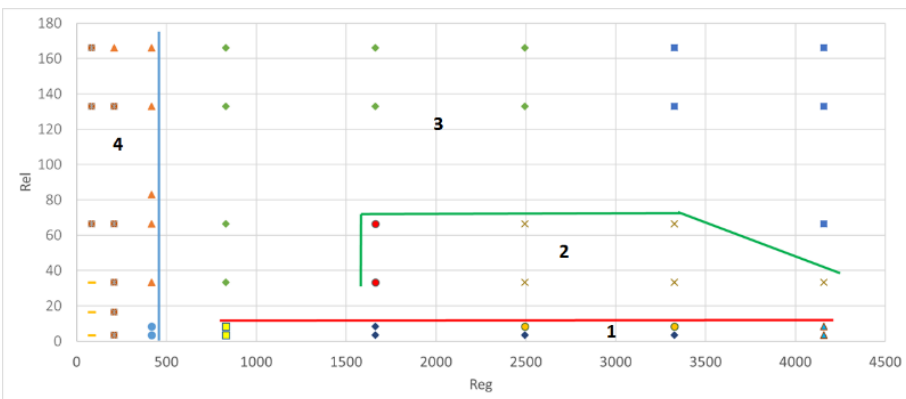

Fig. 5. Flow regime map representing varieties of the geometry of the nitrogen driven rivulet flow of water in the minichannel. 1 - pulsating flow regime, 2 - tapered flow regime, 3 - straight flow regime, 4 - rivulet bridge.

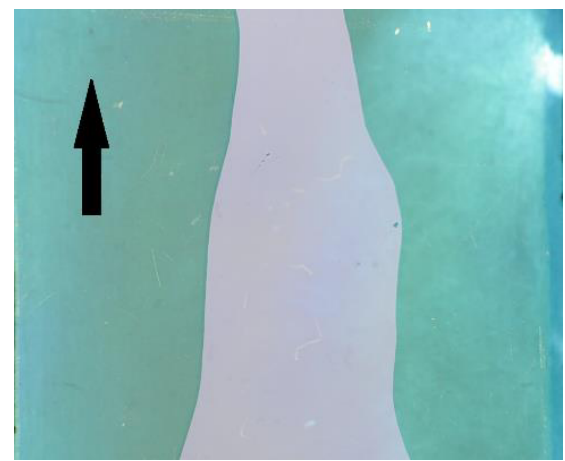

Fig. 6. The nitrogen driven water rivulet flow regime in the minichannel-rivulet bridge.

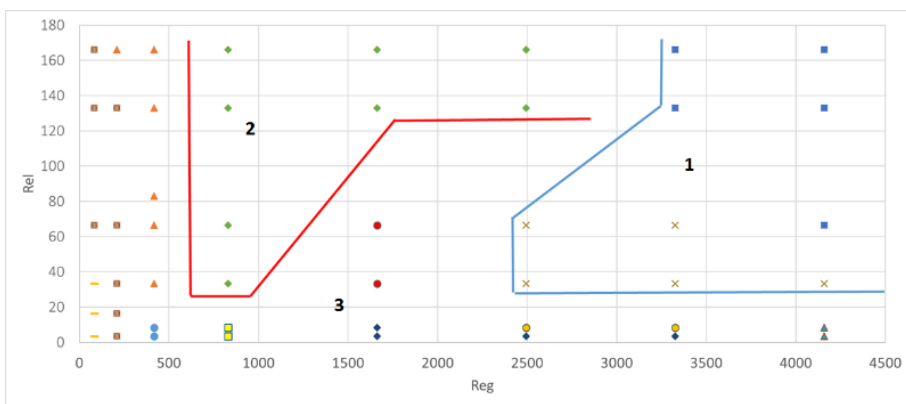

Fig. 7. Flow regime map representing varieties of deformation of the nitrogen driven water rivulet surface in the minichannel: 1 - braiding, 2 - wave, 3 -without any surface deformation. 


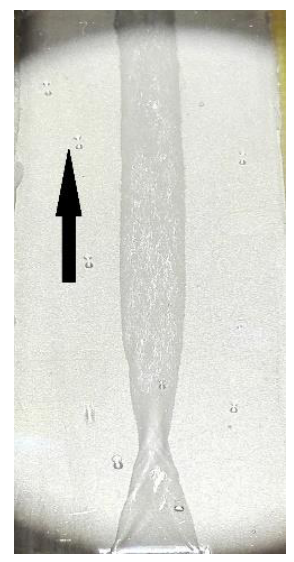

Fig. 8. Braiding surface deformation regime.

This study was performed in the framework of the Russian Science Foundation grant (Agreement No. 14-19-01755).

\section{References}

1. P. Stephan, c. Brandt, Heat Transfer Edging 25, 78 (2004)

2. M. Potash, P.C. Wayner, Int. J. Heat Mass Transfer 15, 1851 (1972)

3. V.V. Kuznetsov, O.V. Vitovskii, V.A. Krasovskii, High Temp 45, 68 (2007)

4. V.V. Cheverda, A. Glushchuk, P. Queeckers, S.B. Chikov, O.A. Kabov, Microgravity sci. tec. 25, 73 (2013)

5. M.V. Bartashevich, O.A. Kabov, V.V. Kuznetsov, Fluid dyn. 45, 924 (2010)

6. P. Schmuki, M. Laso, J. Fluid Mec. 215, 125 (1990)

7. S.V. Alekseenko, A.V. Bobyleb, S.M. Kharlamov, D.M. Markovich, Experimental investigation of heat transfer in a rivulet on the inclined foil (Lisabon, 2008)

8. K. Mertens, V. Putkaradze, P. Vorobieff, J. Fluid Mec. 531, 49 (2005) 